\title{
Design of Two-Channel Low-Delay FIR Filter Banks Using Constrained Optimization
}

\author{
Robert Bregović and Tapio Saramäki \\ Signal Processing Laboratory, Tampere University of Technology, Tampere, Finland
}

\begin{abstract}
This paper shows the efficiency of using constrained optimization for designing two-channel low-delay finite impulse response filter banks. The filter banks under consideration are quadrature mirror filter (QMF) banks and perfect reconstruction (PR) biorthogonal filter banks. The design problems for both types of banks are stated as constrained minimization problems in forms that enable us to minimize the maximum of the stopband energies of the analysis filter(s) subject to the given passband and transition band constraints of the filter(s) as well as subject to the given allowable reconstruction error for QMF banks or the PR property for biorthogonal filter banks. For solving the given optimization problems a modified Dutta-Vidyasagar optimization technique has been used. The efficiency of the proposed design methods is illustrated by means of some examples.
\end{abstract}

Keywords: FIR filter banks, low-delay, two-channel, optimization, QMF, biorthogonal

\section{Introduction}

During the last two decades, filter banks have found various applications in many different areas such as speech coding, scrambling, image compression, and transmission of signals through channels of different bandwidths [1][3]. The main idea of using filter banks is the ability of the system to separate in the frequency domain the signal under consideration into two or more signals or to compose two or more different signals into a single signal. Due to the filtering operations that are performed on the signal passing through the filter bank, a delay between the output and the input signals is introduced. In many applications, it is desirable to keep this delay as short as possible. When using linear-phase finite impulse re- sponse (FIR) filters in synthesizing filter banks, good filter properties result in many cases in high filter orders and in an intolerably high delay. In these applications, low-delay FIR filter banks have to be used. These filter banks are characterized by the property that the orders of the nonlinear-phase building-block FIR filters can be increased to improve the filter properties without increasing the overall delay.

For constructing low-delay FIR filter banks, quadrature mirror filter (QMF) banks and perfect reconstruction (PR) filter banks have been used. For the low-delay QMF banks [4]-[6], the analysis filters are quadrature mirror filters and the PR property cannot be achieved, whereas for PR biorthogonal filter banks [6]-[9], these filters are related through the PR property and have different characteristics.

For designing low-delay QMF banks two iterative approaches have been presented by $\mathrm{Xu}, \mathrm{Lu}$, and Antoniou in [4] and [5]. The main drawback in these iterative algorithms is that the transition band ripple of the amplitude response is not automatically controlled and it is not straightforward to find proper values for the parameters used in the procedures to give a satisfactory filter performance in the transition band. The larger the difference between the filter order and the desired filter bank delay, the more difficult the problem is.

Low-delay PR biorthogonal filter banks have been first introduced by Nayebi, Smith, and Barnwell in [7] and [8]. The filter banks resulting when using their optimization technique are 


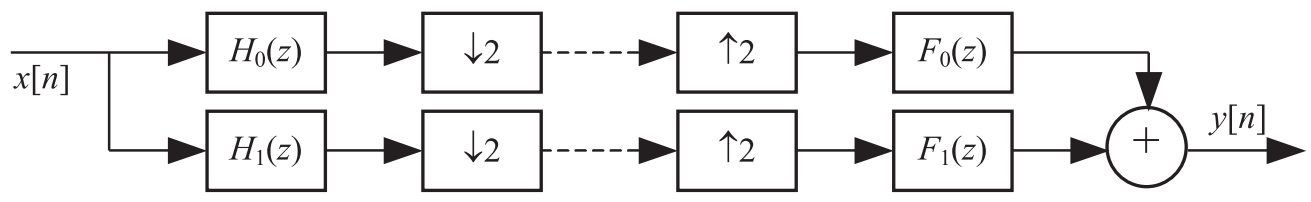

Fig. 1. Two-channel filter bank.

suboptimal, as has been shown in some later papers. However, they have made several important observations concerning the properties of low-delay filter banks that apply to PR biorthogonal filter banks as well as to QMF banks. First, it is not advisable to design filter banks with a very small delay compared to the filter orders, because after a certain filter order for the same overall delay, larger orders result only in a negligible improvement in the performance of the filter bank. Second, additional constraints are usually necessary in the transition bands of the filters due to the possible artifacts occurring in these bands. Abdel-Rahem, El-Guibaly, and Antoniou presented in [9] two approaches for designing low-delay biorthogonal filter banks based on the Lagrange-multiplier method. Filter banks designed by their method have better properties than those designed by Nayebi, Smith, and Barnwell. The method has, like for QMF banks, again problems with the passband and transition band ripples.

This paper shows how the above-mentioned problems can be solved in designing two-channel low-delay FIR filter banks by using constrained optimization. For both QMF banks and PR biorthogonal filter banks, the overall synthesis problem is stated in the form to which the algorithm of Dutta and Vidyasagar [10] can be applied. Several examples are included that illustrate the efficiency of the proposed synthesis scheme in improving the filter bank performance. More details on how to apply the DuttaVidyasagar algorithm to designing filter banks can be found in [11] and [12]. A comprehensive review on two-channel FIR filter banks has been given in [6].

\section{Two-channel filter banks}

This section reviews some basic relations for two-channel filter banks. The block diagram for a two-channel filter bank is shown in Fig. 1 [2].
It consists of an analysis filter bank followed by downsamplers, upsamplers, and a synthesis bank.

It is well known that the relation between the output and input of this system is expressible as

$$
Y(z)=T(z) X(z)+A(z) X(-z),
$$

where the terms

$$
T(z)=\frac{1}{2}\left[H_{0}(z) F_{0}(z)+H_{1}(z) F_{1}(z)\right]
$$

and

$$
A(z)=\frac{1}{2}\left[H_{0}(-z) F_{0}(z)+H_{1}(-z) F_{1}(z)\right]
$$

are the distortion transfer function and the aliasing transfer function, respectively. The second term can be made zero by selecting the synthesis filters as $F_{0}(z)=2 H_{1}(-z)$ and $F_{1}(z)=$ $-2 H_{0}(-z)$. In this case, the residual filter bank distortion becomes

$$
T(z)=H_{0}(z) H_{1}(-z)-H_{1}(z) H_{0}(-z) .
$$

To simplify the overall design problems to be described in the following sections, we use, instead of $H_{0}(z)$ and $H_{1}(z)$, the following transfer functions $[6]$ :

$$
\begin{gathered}
G_{0}(z)=\sum_{n=0}^{N_{0}} g_{0}[n] z^{-n} \\
\equiv H_{0}(z)=\sum_{n=0}^{N_{0}} h_{0}[n] z^{-n} \\
G_{1}(z)=\sum_{n=0}^{N_{1}} g_{1}[n] z^{-n} \\
\equiv H_{1}(-z)=\sum_{n=0}^{N_{1}}(-1)^{n} h_{1}[n] z^{-n} .
\end{gathered}
$$

In the above, $G_{0}(z)$ and $H_{0}(z)$ are identical, whereas $G_{1}(z)=H_{1}(-z)$. Therefore, 


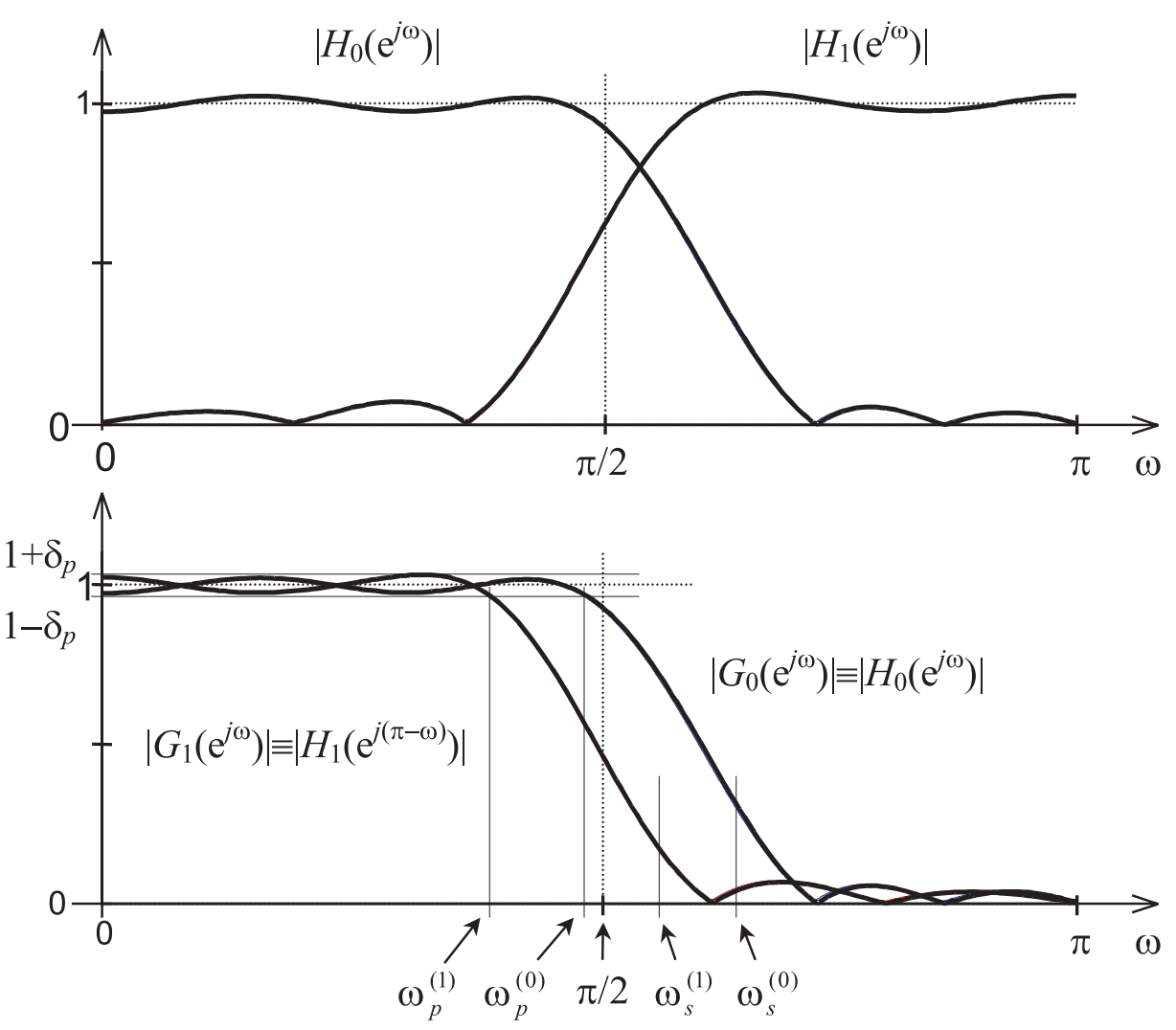

Fig. 2. Specifications for $G_{0}(z)$ and $G_{1}(z)$ as well as the relations between $H_{0}(z)$ and $G_{0}(z)$ and $H_{1}(z)$ and $G_{1}(z)$.

$\left|G_{1}\left(e^{j \omega}\right)\right|=\mid H_{1}\left(e^{j(\pi-\omega)}\right)$ so that the amplitude response of $G_{1}(z)$ is obtained from that of $H_{1}(z)$ by means of the substitution $\pi-\omega \rightarrow \omega$ and vice versa. Fig. 2 exemplifies the above relations in addition to showing the constraints for $G_{0}(z)$ and $G_{1}(z)$ that are used in the problems to be stated in the following sections.

The main advantage of using the above transfer functions $G_{0}(z)$ and $G_{1}(z)$, instead of $H_{0}(z)$ and $H_{1}(z)$, lies in the fact that they are both lowpass filters. This makes their optimization more straightforward as they can be treated in the same way and the synthesis formulas can be expressed in a simplified manner.

\section{Low-delay QMF banks}

In this section, the definition of low-delay QMF banks is given, an appropriate design problem is stated, and an optimization procedure is suggested for solving this problem.

\subsection{Definition of low-delay QMF banks}

The low-delay QMF banks proposed by $\mathrm{Xu}, \mathrm{Lu}$, and Antoniou [4] are characterized by the following properties:

1. $G_{1}(z)=G_{0}(z)=\sum_{n=0}^{N_{0}} g_{0}[n] z^{-n}$, where $N_{0}$ is an odd integer.

2. $\mid G_{0}\left(e^{j \omega} \mid\right.$ approximates zero on $\left[\omega_{s}, \pi\right]$ (stopband) and unity on $\left[0, \omega_{p}\right]$ (passband).

3. $T(z)=\left[G_{0}(z)\right]^{2}+\left[G_{0}(-z)\right]^{2}$ approximates the delay $z^{-K}$ with $K$ is being an odd integer satisfying $K<N_{0}$.

Because of Property 3, the impulse response of $G_{0}(z)$ cannot be symmetric so that all the impulse-response values $g_{0}[n]$ for $n=0,1$, $\ldots, N_{0}$ are unknowns. The reconstruction error given by

$$
\begin{aligned}
& \left|T\left(e^{j \omega}\right)-e^{-j K \omega}\right| \\
& \quad=\left|\left[G_{0}\left(e^{j \omega}\right)\right]^{2}-\left[G_{0}\left(e^{j(\omega+\pi)}\right)\right]^{2}-e^{-j K \omega}\right|
\end{aligned}
$$


is desired to be made small in the overall baseband $[0, \pi]$. Due to the nonlinear-phase characteristics, the performance of $G_{0}(z)$ in the passband and in the transition band must also be controlled.

\subsection{Design method for low-delay QMF banks}

For given $N_{0}, \rho_{s}, \rho_{p}, \delta_{p}$, and $\delta_{a}$ as well as $K$, the problem is to find the $N_{0}+1$ unknown impulse-response coefficients $g_{0}[n]$ of $G_{0}(z)=$ $\sum_{n=0}^{N_{0}} g_{0}[n] z^{-n}$ to minimize

$$
\varepsilon_{0}=\int_{\omega_{s}}^{\pi}\left|G_{0}\left(e^{j \omega}\right)\right|^{2} d \omega
$$

subject to

$$
\begin{gathered}
\max _{\omega \in\left[0, \omega_{p}\right]}|| G_{0}\left(e^{j \omega}\right)|-1| \leq \delta_{p}, \\
\max _{\omega \in\left(\omega_{p}, \omega_{s}\right)}\left|G_{0}\left(e^{j \omega}\right)\right|-1 \leq \delta_{p},
\end{gathered}
$$

and

$$
\begin{gathered}
\max _{\omega \in[0, \pi]} \mid T\left(e^{j \omega}-e^{-j K \omega}\left|=\max _{\omega \in[0, \pi]}\right|\left[G_{0}\left(e^{j \omega}\right]^{2}\right.\right. \\
-\left[G_{0}\left(e^{j(\omega+\pi)}\right)\right]^{2}-e^{-j K \omega} \mid \leq \delta_{a}, \quad(6 \mathrm{c})
\end{gathered}
$$

where

$$
\omega_{p}=\left(1-\rho_{p}\right) \frac{\pi}{2} \quad \text { and } \quad \omega_{s}=\left(1+\rho_{s}\right) \frac{\pi}{2} .
$$

Here the goal is to minimize the stopband energy of the filter under consideration. The first condition of Eq. ( $6 \mathrm{~b})$ forces the maximum passband deviation of the amplitude response from the unity to be less than or equal to $\delta_{p}$, the second condition of Eq. ( $6 \mathrm{~b}$ ) forces the transition band maximum to be less than or equal to $1+\delta_{p}$, and the third condition of Eq. (6c) guarantees that the reconstruction error is smaller than or equal to $\delta_{a}$. The above optimization problem can be solved by properly applying the algorithm of Dutta and Vidyasagar [10] (see, e. g., [11] or [12] for details).

Impulse response for $E(z): K=7, N_{0}+N_{1}=22$
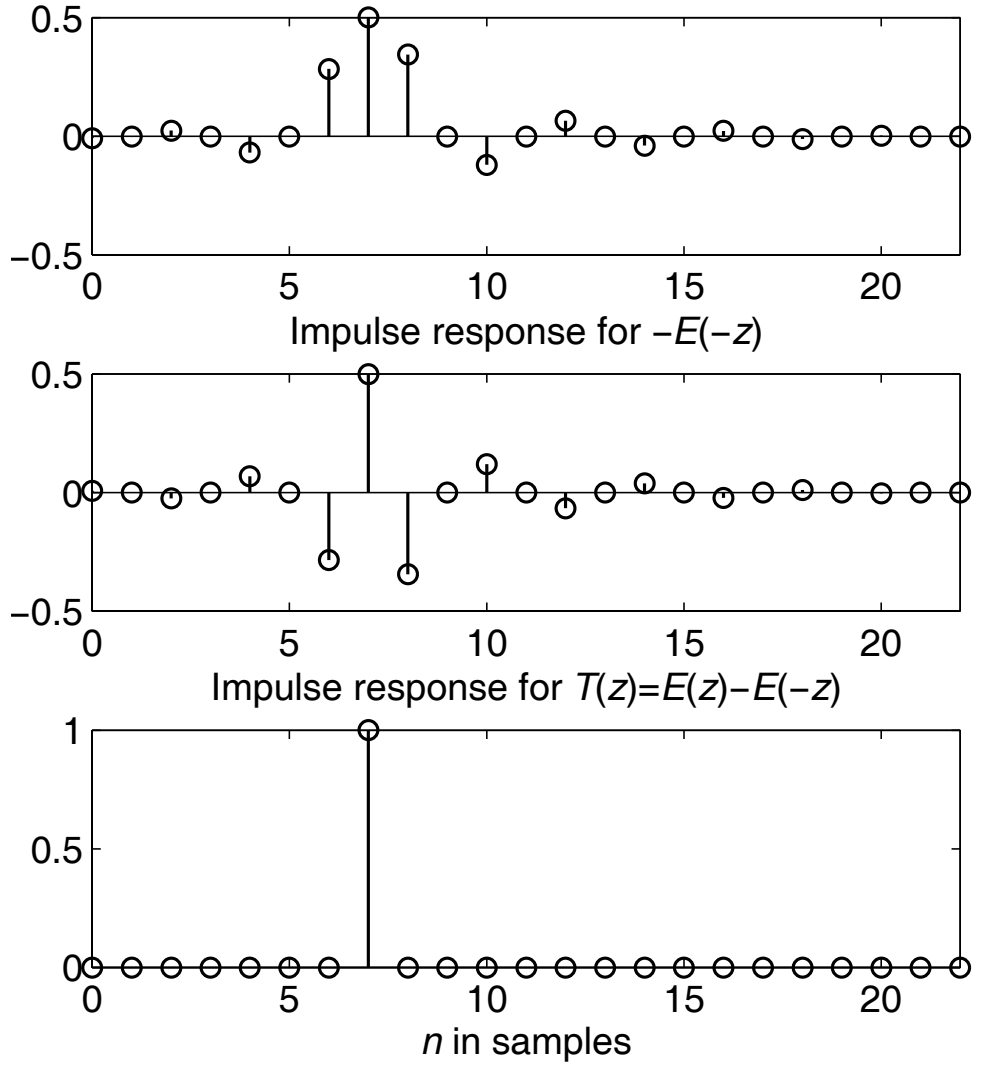

Fig. 3. Impulse responses for $E(z), E(-z)$, and $T(z)$ for a PR filter bank with $K<\frac{N_{0}+N_{1}}{2}$. 


\section{Low-delay PR biorthogonal filter banks}

In this section, the definition of low-delay PR biorthogonal banks is given, an appropriate design problem is stated, and an optimization procedure is suggested for solving this problem.

\subsection{Definition of low-delay PR biorthogo- nal filter banks}

For low-delay PR biorthogonal filter banks $G_{0}(z)$ and $G_{1}(z)$, as given by Eqs. (4a) and (4b), satisfy the following conditions:

1. The impulse responses of $G_{0}(z)$ and $G_{1}(z)$ are not symmetric.

2. The impulse response of $E(z)=G_{0}(z) G_{1}(z)$ $=\sum_{n=0}^{N_{0}+N_{1}} e[n] z^{-n}$ satisfies

$$
e[n]= \begin{cases}\frac{1}{2} & \text { for } n=K \\ 0 & \text { for } n \text { odd and } n \neq K,\end{cases}
$$

where $K$ is an odd integer with $K<\frac{N_{0}+N_{1}}{2}$.

An example for an impulse response of $E(z)$ is shown in Fig. 3. The second condition implies that the overall transfer function between the output and input is $T(z)=E(z)-E(-z)=z^{-K}$ with $K$ less than $\frac{N_{0}+N_{1}}{2}$ (compare Eqs. (3) and (4) and see Fig. 3). The high number of unknowns (altogether $N_{0}+N_{1}+2$ ) and the PR condition with the delay less than half the sum of the filter orders makes the synthesis of the overall system very nonlinear and complicated.

\subsection{Design method for low-delay PR biorthogonal filter bank}

For given $N_{0}, N_{1}, \rho_{p}^{(k)}$ and $\rho_{s}^{(k)}$ for $k=0,1$, and $\delta_{p}$ as well as $K$, the problem is to find the adjustable coefficients of $G_{0}(z)$ and $G_{1}(z)$, as given by Eqs. (4a) and (4b), to minimize

$$
\varepsilon=\max \left(\varepsilon_{0}, \varepsilon_{1}\right)
$$

where

$$
\varepsilon_{k}=\int_{\omega_{s}^{(k)}}^{\pi}\left|G_{k}\left(e^{j \omega}\right)\right|^{2} d \omega \quad \text { for } \quad k=0,1,
$$

subject to

$$
\begin{gathered}
\max _{\omega \in\left[0, \omega_{p}^{(k)}\right]}|| G_{k}\left(e^{j \omega}\right)|-1| \leq \delta_{p}, \text { for } k=0,1, \\
\max _{\omega \in\left(\omega_{p}^{(k)}, \omega_{s}^{(k)}\right)}\left|G_{k}\left(e^{j \omega}\right)\right|-1 \leq \delta_{p} \text { for } k=0,1,
\end{gathered}
$$

and

$$
\begin{gathered}
\max _{\omega \in[0, \pi]} \mid G_{0}\left(e^{j \omega}\right) G_{1}\left(e^{j \omega}\right) \\
-G_{0}\left(e^{j(\omega+\pi)}\right) G_{1}\left(e^{j(\omega+\pi)}\right) \\
-e^{-j K \omega} \mid=0 .
\end{gathered}
$$

Here, $\omega_{p}^{(k)}=\frac{\left(1-\rho_{p}^{(k)}\right) \pi}{2}$ and $\omega_{s}^{(k)}=\frac{\left(1+\rho_{s}^{(k)}\right) \pi}{2}$ for $k=0,1$ are the passband and stopband edges for $G_{0}(z)$ and $G_{1}(z)$ as shown in Fig. 2.

This problem is more complicated than the one stated in Section 3.2. Here the goal is to minimize the maximum of the stopband energies of the two filters under consideration. The condition of Eq. (8d) guarantees that a PR filter bank will be obtained. The above optimization problem can also be solved by properly applying the algorithm of Dutta and Vidyasagar [10] (see, e. g., [11] or [12] for details).

\section{Numerical examples}

This section illustrates, by means of examples, some characteristics of two-channel low-delay QMF banks and low-delay PR biorthogonal filter banks. In addition, the filter banks resulting when applying the proposed optimization scheme are compared to those obtained using other existing techniques.

Example 1. To show the flexibility of the proposed method, two low-delay QMF banks with $N_{0}=31, K=15, \rho_{p}=\rho_{s}=0.19, \delta_{a}=1.38$. $10^{-4}$, and different passband ripples $\delta_{p}=0.087$ and $\delta_{p}=10^{-4}$ have been designed. Fig. 4 shows the amplitude characteristics of the analysis filters as well as the reconstruction errors for both filter banks. As expected, a smaller passband ripple results in a lower stopband attenuation. In both cases, the achieved passband ripple is very small. Only in the transition band, the amplitude characteristic exhibits the value of $1+\delta_{p}$. Moreover, the first filter bank has a performance similar to that of Example 2 in [5]. 


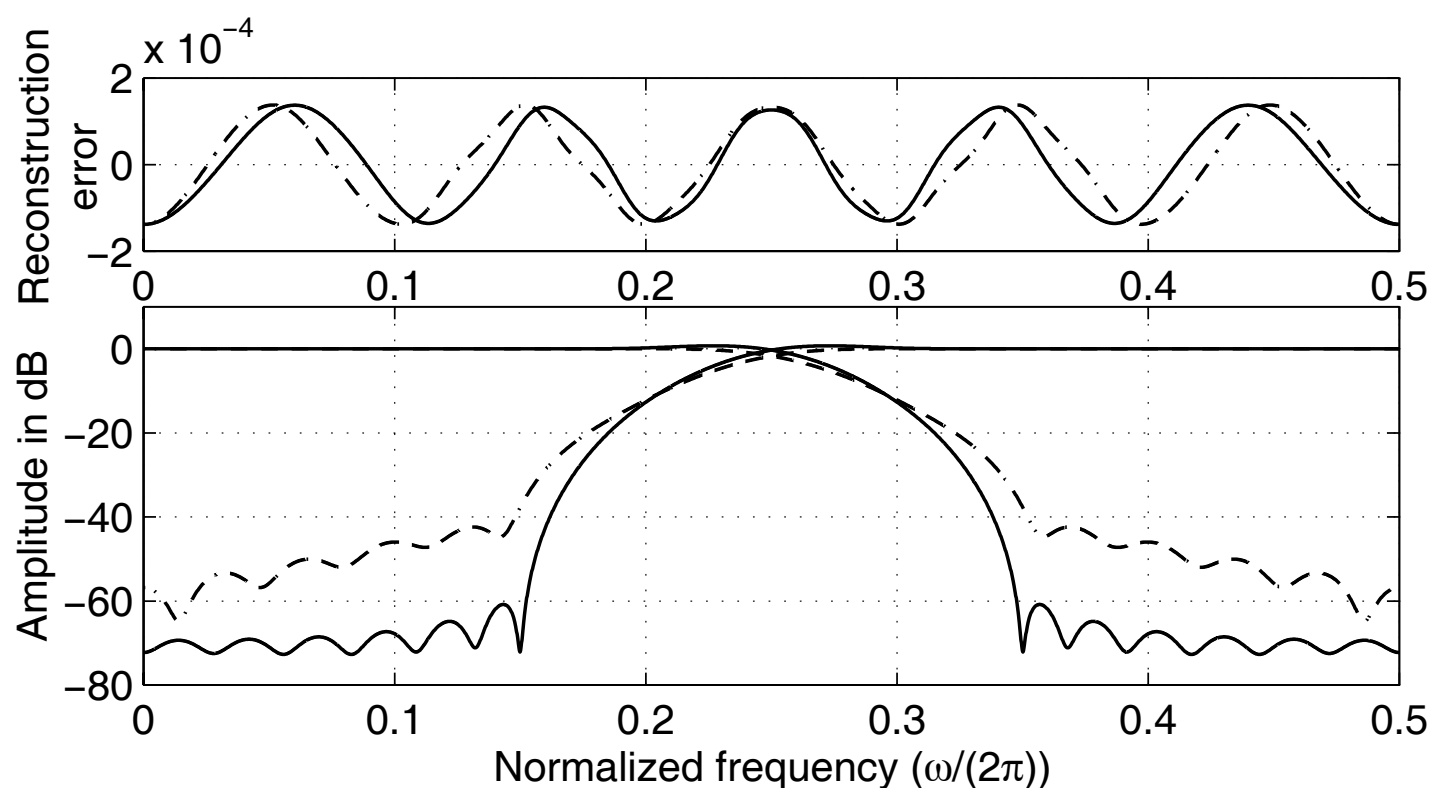

Fig. 4. Low-delay QMF banks with $N_{0}=31$ and $K=15$. The solid and dot-dashed lines show the amplitude responses of analysis filters for $\delta_{p}=0.087$ and $\delta_{p}=10^{-4}$, respectively.

Example 2. It is desired to design a QMF bank with $K=31, \rho_{p}=\rho_{s}=0.172\left(\omega_{s}=\right.$ $0.586 \pi), \delta_{a}=3.23 \cdot 10^{-3}$, and $\delta_{p}=1.6 \cdot 10^{-3}$. Fig. 5 shows the amplitude characteristics of the analysis filters as well as the reconstruction errors for a low-delay QMF bank of order $N_{0}=63$ designed using the proposed method and for a linear-phase QMF bank of order $N_{0}=31$ (Example 1 in [6]). The low-delay filter bank provides significantly better stopband attenuations.
Example 3. A low-delay PR biorthogonal filter bank with $N_{0}=N_{1}=31, K=15, \delta_{p}=$ $3.8 \cdot 10^{-3}$, and $\rho_{p}^{(k)}=\rho_{s}^{(k)}=0.2$ for $k=0,1$ has been designed. The requirements are the same as for the filter bank CLS32-15a in [9]. Fig. 6 shows the amplitude characteristics of the analysis filters for both filter banks. Using the proposed method, filters with considerably better attenuations are obtained.

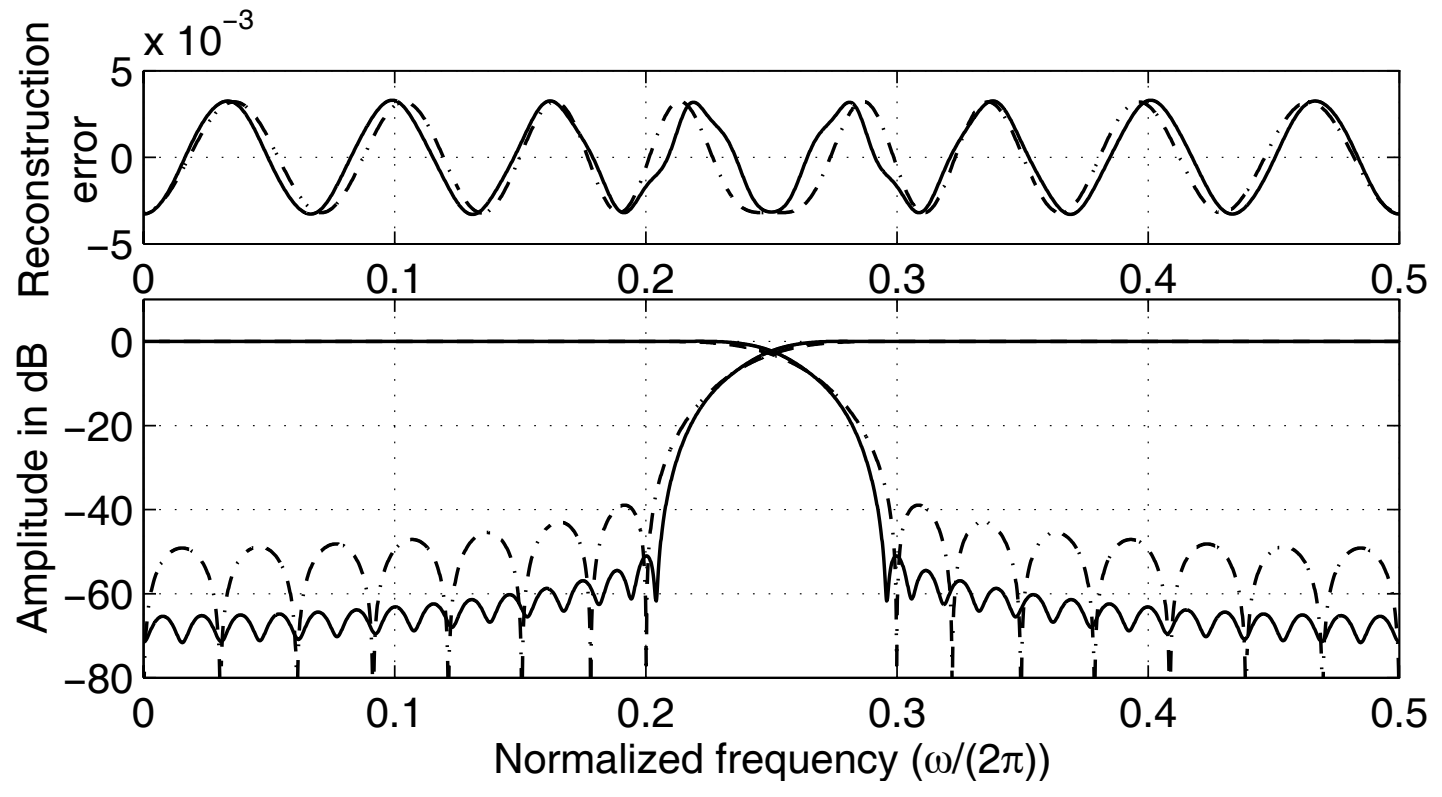

Fig. 5. QMF banks for $K=31$. The solid and dot-dashed lines show the amplitude respones of analysis filters for the low-delay QMF bank for $N_{0}=63$ and the linear-phase QMF bank for $N_{0}=31$, respectively. 


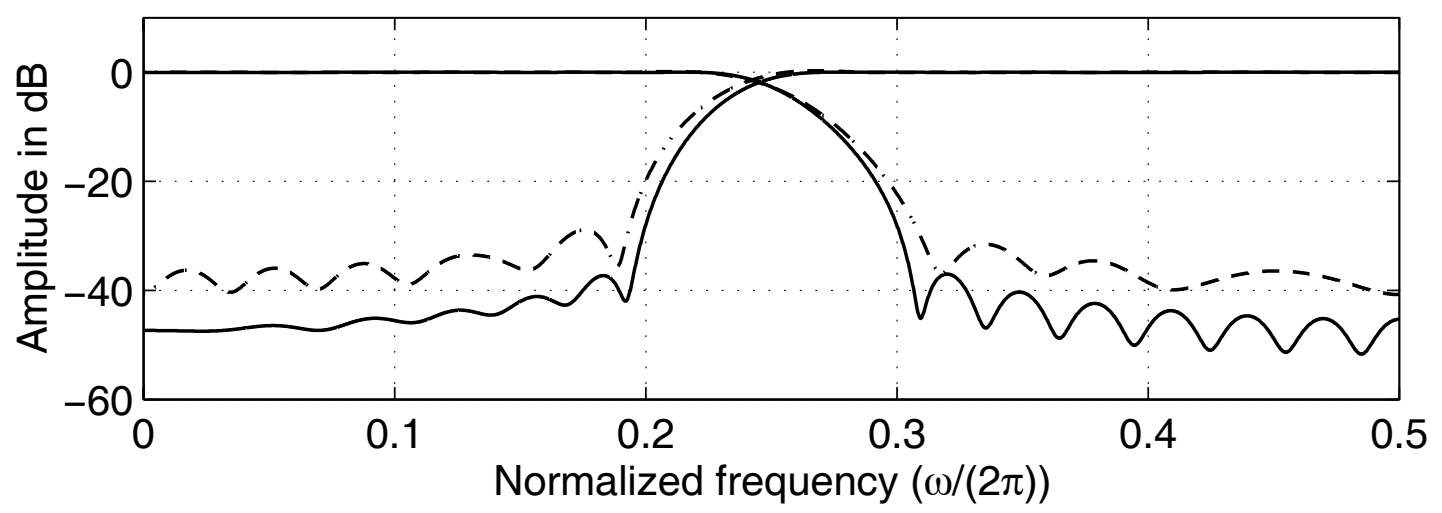

Fig. 6. Low-delay PR biorthogonal filter bank with $N_{0}=N_{1}=31$ and $K=15$. The solid and dot-dashed lines show the amplitude responses of analysis filters for the bank designed by the proposed method and the bank CLS32-15a [9], respectively.

a)
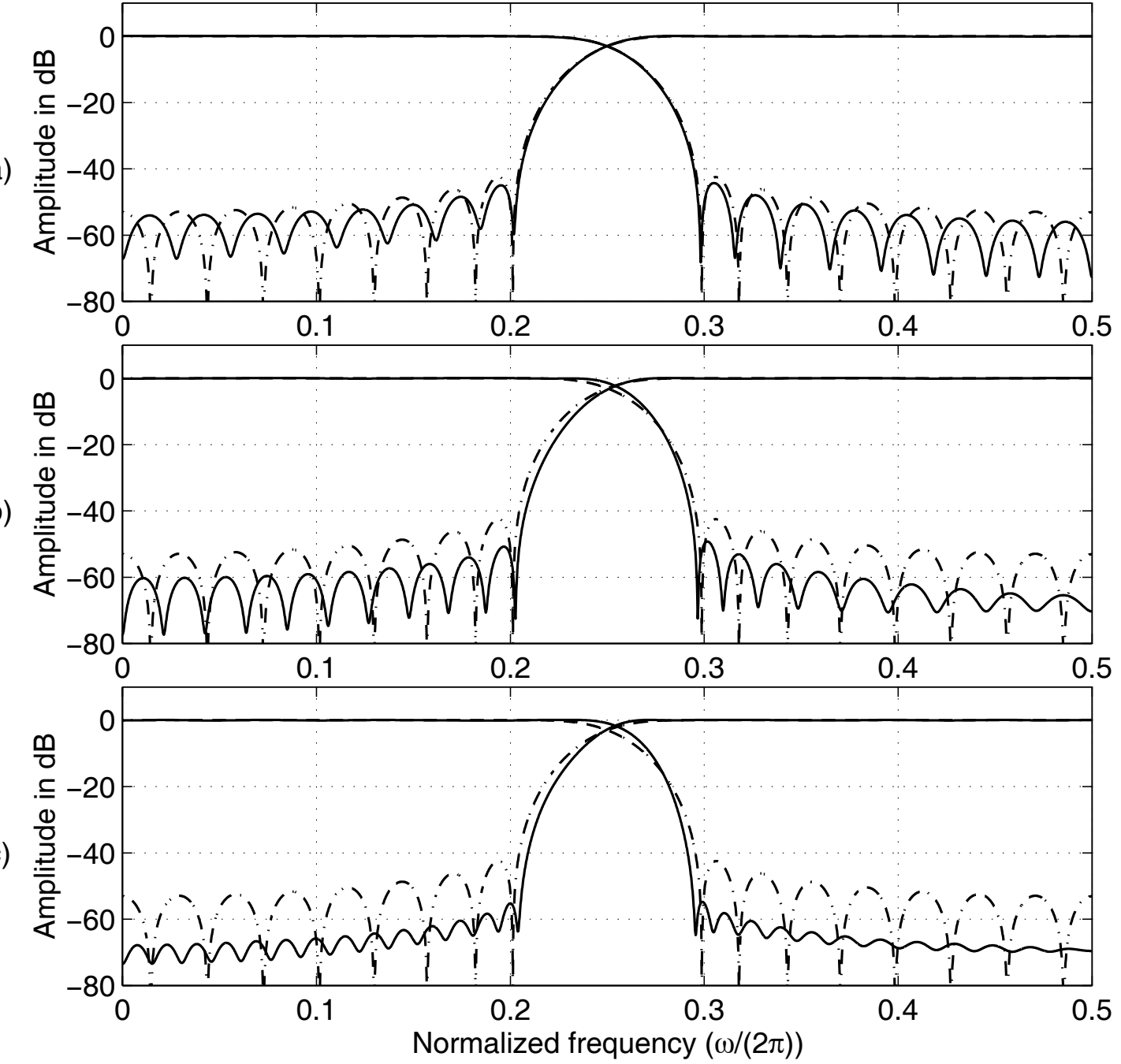

Fig. 7. Comparison for $K=31$ between low-delay PR biorthogonal filter banks with (a) $N_{1}=N_{0}=33$, (b) $N_{1}=N_{0}=45$, (c) $N_{1}=N_{0}=63$ and the linear-phase biorthogonal PR filter bank with $N_{1}=N_{0}=31$. The solid and dot-dashed lines give the amplitude responses of analysis filters for the low-delay banks and the linear-phase bank, respectively. 
Example 4. It is desired to design low-delay PR biorthogonal filter banks for $K=31, \delta_{p}=$ 0.01 , and $\rho_{p}^{(k)}=\rho_{s}^{(k)}=0.172$ for $k=0,1$. The passband and stopband edges for both $G_{0}(z)$ and $G_{1}(z)$ are thus located at $\omega_{p}=0.414 \pi$ and $\omega_{s}=0.586 \pi$ respectively. Fig. 7 compares the optimized low-delay PR filter banks of orders $N_{1}=N_{0}=33, N_{1}=N_{0}=45$, and $N_{1}=N_{0}=63$, with a biorthogonal filter bank with linear-phase subfilters of orders $N_{1}=N_{0}=31$. As can be expected, the stopband attenuations of the analysis filters in the low-delay filter banks increase as the filter orders are made higher.

\section{References}

[1] H. S. Malvar, Signal Processing with Lapped Transforms. Norwood: Artec House, 1992.

[2] P. P. VAIDYANATHAN, Multirate Systems and Filter Banks. Englewood Cliffs, N. J.: Prentice Hall, 1993.

[3] N. J. FLIEGE, Multirate Digital Signal Processing Period, Chicester: John Wiley and Sons, 1994.

[4] H. XU, W.-S. LU AND A. ANTONIOU, "An improved method for the design of FIR quadrature mirrorimage filter banks," IEEE Trans. Signal Processing, vol. 46, pp. 1275-1281, May 1998.

[5] W.-S. LU, H. XU AND A. ANTONIOU, "A new method for the design of FIR quadrature mirror-image Filter Banks," IEEE Trans. Circuits Syst. II, vol. 45, pp. 922-926, July 1998.

[6] R. BREGOVIĆ AND T. SARAMÄKI, "Two-channel FIR filter banks - A tutorial review and new results," in Proc. Second Int. Workshop on Transforms and Filter Banks, Brandenburg, Germany, March 1999, TICSP \#4, pp. 507-558.

[7] K. NAYEBI, T. P. BARNWELl III AND M. J. T. SMITH, "Time-domain filter bank analysis: A new design theory," IEEE Trans. Signal Processing, vol. 40, pp. 1412-1429, June 1992.

[8] K. NAYEBI, T. P. BARNwELl III AND M. J. T. SMITH, "Low delay FIR filter banks: Design and evaluation," IEEE Trans. Signal Processing, vol. 42, pp. 24-31, Jan. 1994.

[9] E. AbDEl-RAHEem, F. El-Guibaly AND A. ANTONIOU, "Design of low-delay two-channel FIR filter banks using constrained optimization," Signal Procsssing, vol. 48, pp. 183-192, Feb. 1996.

[10] S. R. K. DUTTA AND M. VidYASAGAR, "New algorithms for constrained minimax optimization," Mathematical programming, vol. 13, pp. 140-155, 1977.
[11] R. BREGOVIĆ AND T. SARAMÄKI, “A generalpurpose optimization technique for designing twochannel FIR filter banks," in Proc. European Signal Processing Conference, EUSIPCO-2000, Tampere, Finland, Sept. 2000, vol. I, pp. 369-372.

[12] T. SARAMÄKI, "A generalized class of cosinemodulated filter banks," in Proc. First Int. Workshop on Transforms and Filter Banks, Tampere, Finland, Feb. 1998, pp. 336-365.

Received: October, 2000 Accepted: November, 2000

Contact address:

Robert Bregović and Tapio Saramäki Signal Processing Laboratory Tampere University of Technology P. O. Box 553, FIN-33101 Tampere, Finland e-mail: bregovic@cs.tut.fi and ts@cs.tut.fi

ROBERT BREGOVIć was born in Varaždin, Croatia, in 1970. He has received the degrees of Diploma Engineer and Master of Science in electrical engineering from Faculty of Electrical Engineering and Computing, University of Zagreb, Croatia, in 1994 and 1998, respectively.

From 1994 to 1998 he worked as an assistant in the Department of Electronic Systems and Information Processing of the same Faculty. In 1999 he was a visiting researcher at the Tampere International Center for Signal Procesing. Since January 2000 he has been a researcher at the Signal Processing Laboratory of Tampere University of Technology working towards his doctoral degree.

His research interests include multirate signal processing, digital filter banks, optimization procedures for digital filter design and implementation of algorithms on digital signal processors. Robert Bregové is a member of IEEE Signal Processing Society and Croatian Society KoREMA.

TAPIO SARAMÄKI was born in Orivesi, Finland, on June 12, 1953. He has received the degrees of Diploma Engineer (with honors) and Doctor of Technology (with honors) in electrical engineering from the Tampere University of Technology, Tampere, Finland, in 1978 and 1981, respectively.

Since 1977, he has held various research and teaching positions at Tampere University of Technology, where he is currently a Professor of Signal Processing and a Docent of Telecommunications. He is also a co-founder and a system-level designer of VLSI Solution Oy, Tampere, Finland, specializing in VLSI implementations of sigma-delta modulators and signal signal processing algorithms for various applications. Currently, Dr. Saramäki is an Associate Editor for IEEE Transactions on Circuits and Systems - II: Analog and Digital Signal Processing. In 1982, 1985, 1986, 1990, and 1998 he was a Visiting Research Fellow at the University of California at Santa Barbara.

Dr. Saramäki has written more than 200 international journal and conference articles, six international book chapters, and holds three patents. His research interests are in digital signal processing, especially filter and filter bank design, VLSI implementations, and communications applications, as well as approximation and optimization theories.

Dr. Saramäki received the 1987 Guillemin-Cauer Award for the best paper of the IEEE Transactions on Circuits and Systems. He is a founding member of the Median-Free Group International. 